\title{
INEQUALITIES ARISING FROM GENERALIZED EULER-TYPE CONSTANTS MOTIVATED BY LIMIT SUMMABILITY OF FUNCTIONS
}

\author{
Mohammad Hadi Eghtesadi FARd And M. H. Hooshmand
}

Abstract. Limit summability of real functions was introduced by M.H. Hooshmand in 2001. In order to study derivation of the limit summand function, he has introduced a functional sequence corresponding to a given function $f$ with $D_{f} \supseteq \mathbb{N}^{*}$ that is related to the Euler-type constants. In the way, we prove two main criteria for its convergence together with an extensive inequality between the limit summand function and the generalized Euler-type constants. The main inequality is also extended whenever $f$ is a convex or concave function. Among other things, we obtain some inequalities for many special functions such as the gamma, digamma and zeta functions.

Mathematics subject classification (2010): 40A30, 39B62, 26D07, 26D10, 33E20.

Keywords and phrases: Convex and concave functions, inequalities involving functions, limit summability of real functions, Euler-type constants, gamma function.

\section{REFERENCES}

[1] T. M. Apostol, Introduction to Analytic Number Theory, Springer, 1976.

[2] E. Artin, The Gamma Function, Holt Rhinehart \& Wilson, New York, Transl. by M. Butler (1931) from Einfuhrung un dfr Theorie der Gamma fonktion, Teubner, Leipzig, 1964.

[3] M. H. Hooshmand, Limit Summability of Real Functions, Real Analysis Exchange, 27(2)(2002), 463-472.

[4] M. H. Hooshmand, Another Look at the Limit Summability of Real Functions, J. Math. Ext., 4(2009), 73-89.

[5] M. H. Hooshmand, Analytic Summability of Real and Complex Functions, J. Contemp. Math. Anal., 4(2016), 63-73.

[6] A. Laforgia And P. Natalini, Exponential, Gamma and Polygamma Functions Simple Proofs of Classical and New Inequalities, J. Math. Anal. Appl., 407(2)(2013), 495-504.

[7] M. Muller, D. SChleicher, Fractional Sums and Euler-Like Identities, Ramanujan J., 21(2)(2010), 123-143.

[8] J. SANDOR, On Generalized Euler Constants and Schlomilch-Lemonnier Type Inequalities, J. Math. Anal. Appl., 328 (2007), 1336-1342.

[9] R. J. Webster, Log-Convex Solutions to the Functional Equation $f(x+1)=g(x) f(x)$ : $\Gamma$-Type Functions, J. Math. Anal. Appl., 209 (1997), 605-623. 